no siendo necesario el tratamiento con radioterapia externa.

El pronóstico de los pacientes con enfermedad metastásica en la región orbitaria es desfavorable. Los estudios publicados en cuanto a la supervivencia son variables, estimándose entre 7,5-30 meses. En nuestro caso fue de 10 meses.

\section{CONCLUSIÓN}

Consideramos que el cáncer de próstata debería ser tenido en cuenta a la hora del diagnóstico diferencial de masas retrorbitarias de posible origen metastásico en pacientes con clínica neuro-oftalmológica, aún cuando el paciente no relate clínica urológica específica.

\section{BIBLIOGRAFIA Y LECTURAS RECOMENDADAS ( ${ }^{*}$ lectura de interés $y^{* *}$ lectura fundamental)}

1. PIERAS, E.; ROSALES, A.; LÓPEZ, H. y cols.: "Metástasis intracraneales en cáncer de próstata". Actas Urol. Esp., 24: 626, 2000.

2. FRANCO, E.; GIL-NÉCIJA, E.; CANO, G. y cols.: "Metástasis esfenoidal simulando meningioma como manifestación inicial de adenocarcinoma de próstata". Rev. Neurol., 29: 929, 1999.

3. BONILLO GARCÍA, M.A.; QUEIPO ZARAGOZÁ, J.A.; PALMERO MARTÍ, J.L. y cols.: "Metástasis retroorbitaria como manifestación inicial del cáncer de próstata". Actas Urol. Esp., 27: 562, 2003.

**4. SUTTON, M.A.; WATKINS, H.L.; GREEN, L.K. y cols.: "Intracranial metastases as the first manifestation of prostate cancer". Urology, 48: 789, 1996.

5. PRAT-BARTOMEU, J.: "Metástasis orbitarias en el adulto". Rev. Neurol., 31: 1261, 2000.

**6. BALTOGIANNIS, D.; KALOGEROPOULOS, C.; IOACHIM, E. y cols.: "Orbital metastasis from prostatic carcinoma”. Urol. Int., 70: 219, 2003.

7. GONZÁLEZ, C.; KUZEL, T.; CARTER, M.: "Metastatic adenocarcinoma of the prostate to the orbit as a presenting symptom". J. Urol., 157: 625, 1997.

8. BATSON, O.V.: "The function of the vertebral veins and their role in the spread of metastases". Annals of Surgery, 112: 138, 1940

9. AUBERT, J.; IRANI, J.: "Saint-Blancatp. Orbital metastasis of prostatic cancer. Clinical and therapeutic aspects. A propos of a case". Chirurgie, 121: 672, 1997.

10. CARRIERE, V.M.; KARCIOGLU, Z.A.; APPLE, D.J. y cols.: "A case of prostate carcinoma with bilateral orbital metastases and the review of the literature". Ophthalmology, 89: 402, 1982.

Casos Clínicos

Arch. Esp. Urol., 61, 4 (527-531), 2008

\title{
LINFOMA TESTICULAR PRIMARIO. PRESENTACIÓN DE DOS CASOS Y REVISIÓN DE LA LITERATURA
}

\author{
Paloma Delgado Bavai, Jorge Abad Roger, Araceli \\ Bono Ariño, Miguel Esclarin Duny, Miguel Marigil \\ Gomez y José Ignacio Sanz Velez.
}

Servicios de Urología y Anatomía Patológica'. Hospital San Jorge. Huesca. España.

Resumen.- OBJETIVO: Comunicación de dos casos clínicos con diagnóstico de linfoma testicular primario y revisión de la literatura existente sobre esta patología.

MÉTODOS/RESULTADOS: Descripción de dos casos de linfoma testicular primario en dos varones de 69 y 61 años. En ambos casos su motivo de consulta fue un aumento de volumen testicular derecho. Tras practicar una orquiectomía inguinal se diagnóstico, en el primer caso, un linfoma difuso tipo $T$ de células grandes y medianas, estadio I (E)-A, que tras seguir tratamiento con quimioterapia (R-CHOP) se mantiene en remisión a los tres años. En el segundo caso, el diagnóstico fue de linfoma difuso de células grandes

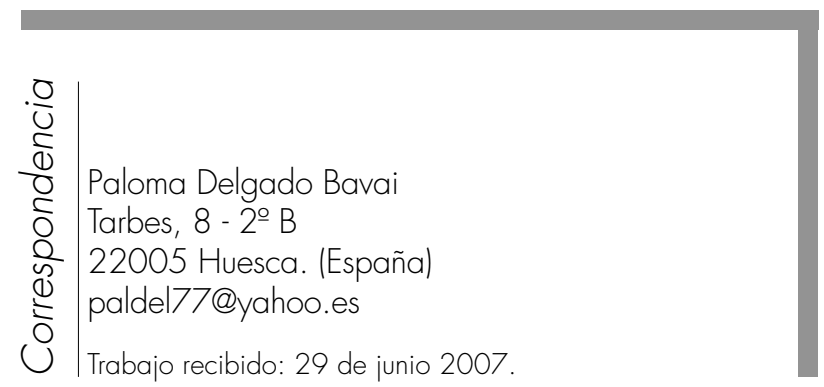


tipo B, que tras recibir distintas pautas de quimioterapia, falleció a los 14 meses del diagnóstico.

CONCLUSIONES: El linfoma testicular es una entidad muy poco frecuente, a pesar de ser considerado el tumor testicular más común en mayores de 60 años. En su mayoría se trata de linfomas no Hodgkin difusos de grado intermedio alto de malignidad e inmunofenotipo $B$, considerando como excepcionales los de tipo T. Su pronóstico es pobre debido a su gran tendencia a la propagación sistémica. Su tratamiento se basa en orquiectomía, quimioterapia y radioterapia, si bien no existe una pauta estandarizada del mismo.

Palabras clave: Linfoma testicular primario. Cáncer testicular. Linfoma extranodal.

Summary.- OBJECTIVE: We report two cases of primary testicular lymphoma and performed a bibliography review about this pathology.

METHODS/RESULTS: We describe two cases of primary testicular lymphoma in two male patients 69 and 61 yr. old respectively. In both cases, reason for consultation was increase of the right testicular size. After inguinal orchyectomy the diagnosis for the first case was large and medium T cell diffuse lymphoma, stage I (E)-A; three years after chemotherapy (R-CHOP) the patient is in complete remission. In the second case, the diagnosis was diffuse large B-cell lymphoma, and affer receiving various chemotherapy regimens died 14 months after diagnosis.

CONCLUSIONS: Testicular lymphoma is a very rare entity, despite being considered the most common testicular tumor in patients over the age of 60 years. Most of them are nonHodgkin diffuse, intermediate or high grade of malignancy, B-cell immunophenotype, being T-cell exceptional. Prognosis is poor due to their high tendency to systemic dissemination. The treatment is based on orchiectomy, chemotherapy and radiotherapy, although there is not a standardized regimen

Keywords: Primary testicular lymphoma. Testicular cancer. Extranodal lymphoma.

\section{INTRODUCCIÓN}

El cáncer testicular supone el 2-3\% de los tumores del hombre, sin embargo, representa el proceso maligno más frecuente en varones de 15 a 35 años (1) (hasta el $35 \%$ en algunas series). A pesar del marcado aumento de su incidencia en los últimos 40 años, llegando a duplicarse en algunos casos, los porcentajes de mortalidad han disminuido debido a los avances en el tratamiento gracias al uso combinado de quimioterapia, radioterapia y cirugía.

Al analizar la incidencia por países se observa una gran variabilidad geográfica, presentando cifras superiores en los países escandinavos, Nueva Zelanda y Alemania respecto a las de los países asiáticos o África.

Los tumores primarios de células germinales son la estirpe histológica más frecuente de todas las neoplasias testiculares (90-95\%). El linfoma testicular, a pesar de representar tan sólo entre el 1 y el $9 \%$ (según las series) de los tumores de esta localización, es considerado el tumor testicular más común en mayores de 60 años $(2,3)$. Si además, tenemos en cuenta que el linfoma testicular supone el $1 \%$ de los Linfoma No-Hodgkin (LNH)2 $y$ el $2 \%$ de los linfomas extraganglionares primarios, podemos afirmar que nos encontramos ante un proceso muy poco frecuente.

Revisamos la bibliografía existente y presentamos dos casos detectados en nuestra área de salud recientemente.

\section{CASOS CLÍNICOS}

\section{Caso 1}

Paciente de 69 años, que consulta por aumento de volumen en teste derecho, indoloro, de tres meses evolución. A la exploración física se aprecia masa testicular, de consistencia irregular con transiluminación negativa. Como pruebas complementarias destacan un estudio de marcadores para tumores testiculares negativo, imágenes ecográficas nodulares múltiples en testículo derecho y un TAC sin signos sugestivos de adenopatías ni afectación visceral.

Tras la realización de una orquiectomía inguinal y análisis de las muestras obtenidas, se diagnostica de linfoma difuso tipo $T$ (Figuras 1 y 2), de células grandes y medianas en estadío l(E)-A.

Como tratamiento se le administra quimioterapia citorreductiva según esquema R-CHOP, manteniendo su proceso en remisión a los tres años de seguimiento.

\section{Caso 2}

Paciente de 61 años, con antecedentes de prostatectomía radical con linfadenectomía obturatriz de estadiaje por carcinoma de próstata medianamente diferenciado (suma Gleason 5), con afectación microscópica limitada de los márgenes de resección. A los tres meses de la cirugía prostática consulta por un aumento de volumen testicular derecho, con marcadores de fumor testicular negativos, masa con áreas irregulares en ecografía y disminución de flujo vascular en estudio doppler. La eva- 


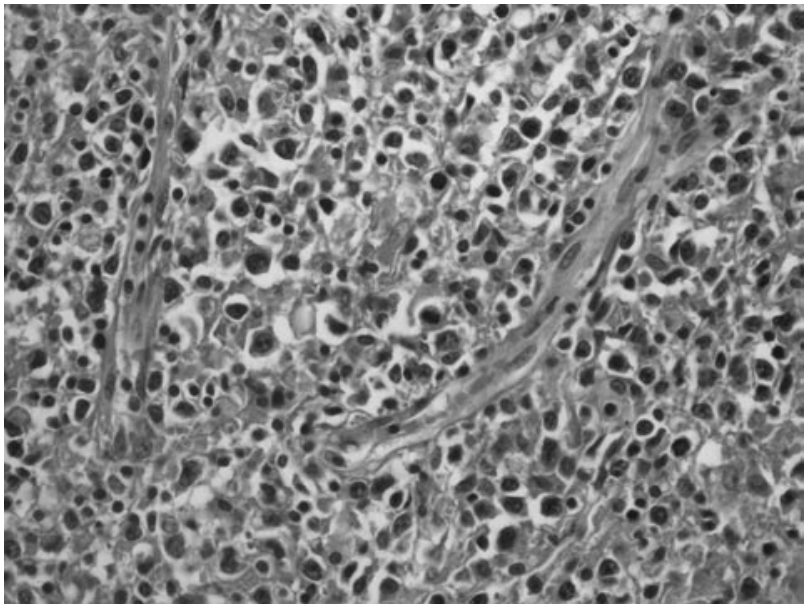

FIGURA 1. Se observa un componente linfoide atípico polimorfo y pleomórfico formado por células pequeñas, medianas y grandes con numeroso mitosis con presencia de numerosos vasos (HE, x400).

luación preoperatoria había incluido un TAC abdominal sin hallazgos. El TAC post orquiectomía detecta adenopatías retroperitoneales desde área inguinal hasta bifurcación de renales.

Tras practicarle una orquiectomía inguinal fue diagnosticado de linfoma testicular difuso de célula grande tipo $B$ (Figuras 3 y 4 ).

Se le administró una primera pauta de quimioterapia citorreductiva según esquema R-CHOPx8, con remisión completa tras la inducción. Presentó una recidiva precoz tratada en segunda línea mediante esquema IFOVM / DHAP, con remisión inicial tras tres ciclos y posteriormente una nueva recidiva, falleciendo a los 14 meses del diagnóstico.

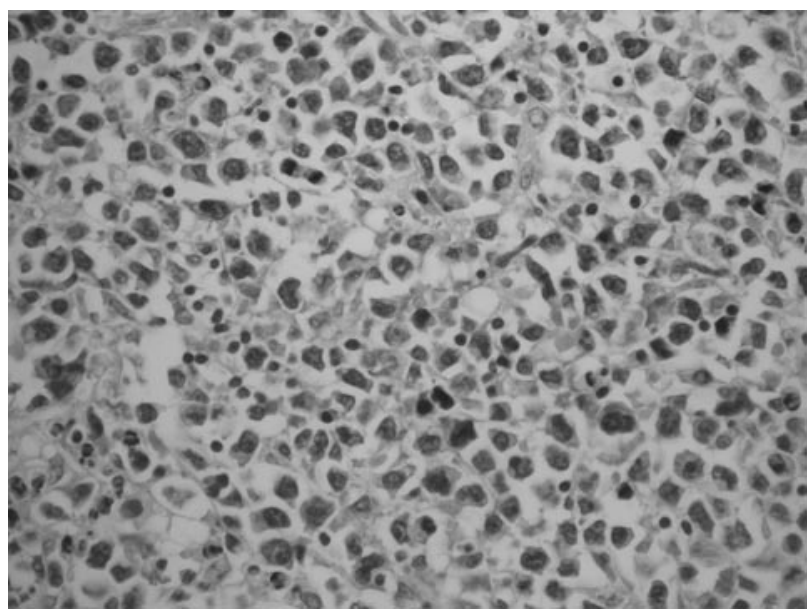

FIGURA 3. Se observa la presencia de células linfoides atípicas de tamaño mediano y grande de núleolo vesiculoso y uno o varios nucleolos (HE, x400).

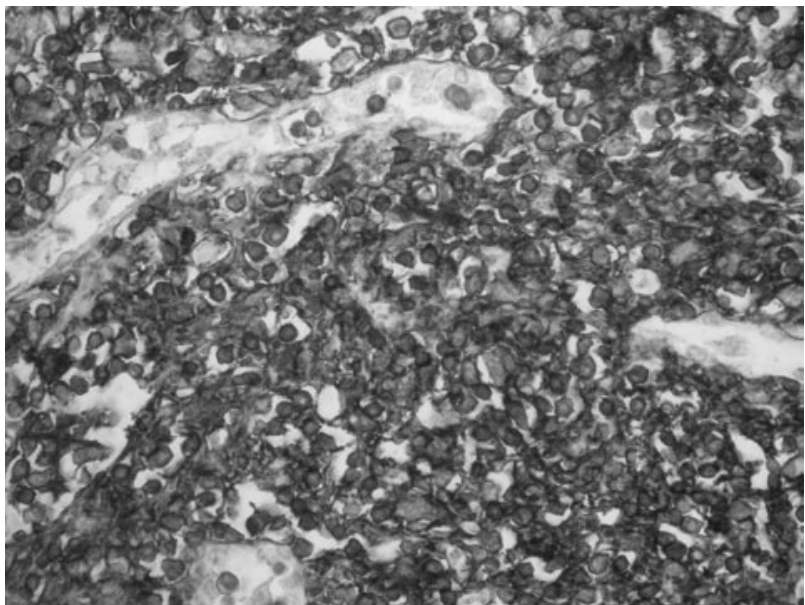

FIGURA 2. Expresividad difusa citoplasmática con CD-3 (X400).

\section{DISCUSIÓN}

El linfoma de testículo representa entre el 1-9\% de los tumores de testículo según las series, el $1 \%$ de los $\mathrm{LNH}$ y el $2 \%$ de los linfomas extraganglionares primarios (LEP). Por LEP entendemos las neoplasias linfoides que se originan en estructuras extraganglionares, admitiendo la participación de grupos ganglionares vecinos.

Se trata de la forma más frecuente de cáncer testicular en varones mayores de 60 años (25-50\%) (2) aunque, de modo excepcional, también se han descrito casos en niños (4).

Se considera el tumor testicular secundario más frecuente para cualquier grupo de edad, sin olvidar que el

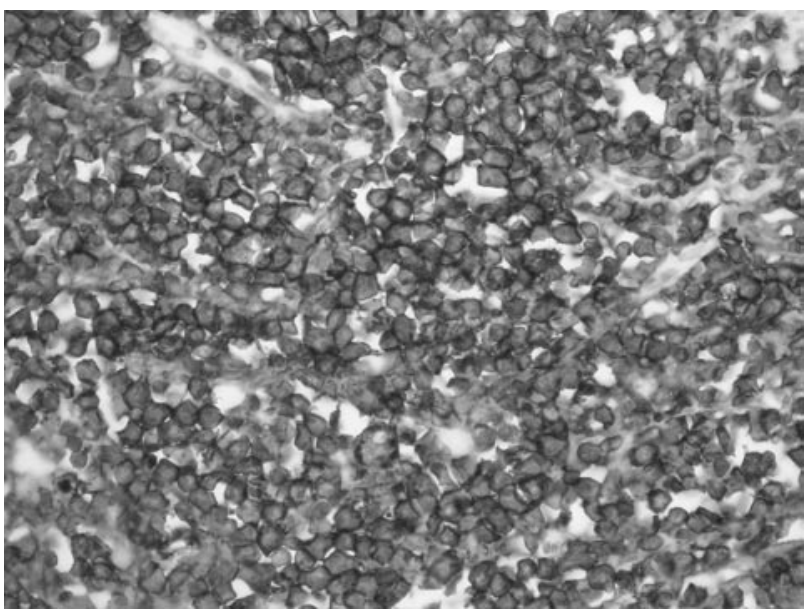

FIGURA 4. Expresividad intensa y difusa citoplasmática con CD-20 (x400). 
testículo es el primer lugar de recidiva de la leucemia linfoide en niños.

La etiología del linfoma testicular primario es desconocida, aunque en su génesis se le ha relacionado con episodios de orquitis crónica, traumatismos, criptorquidia o filariasis (2). En el linfoma testicular, el $20 \%$ de los casos, son o llegan a ser bilaterales, bien de un modo sincrónico o asincrónico (más frecuentemente), considerando esto como un signo de mal pronóstico (3).

También parece existir relación con alteraciones de la inmunidad, aumentando su incidencia (al igual que sucede con otros tumores) en trasplantados y en pacientes afectados de SIDA, en los que la incidencia se eleva hasta el $25 \%$ de las neoplasias testiculares $(3,5)$. Recientemente se ha descrito algún caso relacionado con el uso prolongado de azatioprina.

En los últimos años se están estudiando distintas alteraciones genéticas relacionadas con la aparición de tumores testiculares en general (presencia de isocromosoma en el brazo corto del cromosoma 12...) y del linfoma testicular en particular (delección del brazo largo del cromosoma 6) así como su posible valor pronóstico. En este sentido, también se han abierto diferentes líneas de investigación sobre la relación de estos tumores y posibles alteraciones del HLA (delecciones del HLA I y HLA II DR/DQ) la expresión de determinadas sustancias por parte de las células tumorales (desadherina) y otros desórdenes moleculares (hipermetilación de BRCA1, $\mathrm{VHL}$...).

Macroscópicamente, el aspecto del linfoma testicular suele ser el de una masa carnosa, blanco-grisácea con focos extensos de necrosis y hemorragia, que desplaza de modo difuso al parénquima testicular, aunque habitualmente respeta la vaginal. De difícil diferenciación con el seminoma, si bien en éste es menos frecuente la extensión a estructuras extratesticulares. Microscópicamente, es común observar invasión vascular.

Desde un punto de vista histopatológico, en su mayoría se trata de linfoma no Hodgkin difuso de grado intermedio-alto de malignidad e inmunofenotipo $B(6)$, aunque de modo excepcional se han descrito casos de linfoma de células T (Natural Killer) (7) incluso en pacientes pediátricos, todos ellos de gran agresividad.

Analíticamente la LDH está elevada, y aunque no es un marcador específico, su valor sí está directamente relacionado con la actividad y volumen tumoral. Otros marcadores tumorales relacionados con otras estirpes de tumores testiculares como la Alfafetoproteína, la BHCG o el CEA, no parecen estar elevados en el linfoma testicular $(2,8)$.

El diagnóstico diferencial lo planteamos con entidades como el seminoma anaplásico, basándonos principalmente en marcadores inmunohistoquímicos específicos como el Ag Común Leucocitario o Ac PanB, la orquitis granulomatosa, brucelosis y tuberculosis testicular, rabdomiosarcoma, leucemia, sarcoma granulocítico, hiperplasia linfoide, pseudolinfoma testicular y metástasis de tumores extratesticulares.

El pronóstico del linfoma testicular es pobre debido a una gran tendencia a la propagación sistémica temprana, afectando áreas extraganglionares como el anillo de Waldeyer, Sistema Nervioso Central, médula ósea, pulmón y piel $(2,6,8)$. Aún así, el $50 \%$ de pacientes se diagnostican en estadío IE (estrictamente localizado) de Ann Arbor. La supervivencia global a los 5 años se aproxima al $50 \%$ y la supervivencia libre de enfermedad es del $40 \%$ (6). Como factores de mal pronóstico se consideran, fundamentalmente, el estadio tumoral, la edad (peor cuanto más avanzada), la afectación bilateral o de estructuras adyacentes (epidídimo, cordón o vasos), la presencia de síndrome constitucional o síntomas $B(2,6)$ y el grado de esclerosis, según estudios, el linfoma con esclerosis mostró un mejor pronóstico que los que carecían de esta característica. La variante histológica tiene una importancia relativa a la hora de establecer el pronóstico ya que la mayoría son de alto grado de malignidad.

Su tratamiento se sustenta en tres pilares fundamentales: orquiectomía, quimioterapia y radioterapia, si bien, dada su poca frecuencia, no existe un tratamiento estandarizado del mismo.

En la mayoría de las series se demuestra que el tratamiento con 6 ciclos de quimioterapia, R-CHOP o B$\mathrm{CHOP}$, aumenta el porcentaje de remisiones completas, sin embargo, en el caso de niños con linfoma testicular localizado, ésta podría ser prescindible al ser menos probable la asociación con enfermedad sistémica oculta. En general, el número de ciclos y la intensidad del tratamiento con quimioterapia dependerán de los factores pronósticos iniciales (6). A pesar del beneficio que supone su administración como tratamiento coadyuvante, la orquiectomía sigue siendo necesaria independientemente de su aplicación, dada la quimioresistencia que presentan en ocasiones estos tumores, explicada, en parte, por la expresión de glicoproteína (P-gp) y proteínas (MRP-1) resistentes a estas drogas por parte de los mismos.

No existe consenso respecto al uso de radioterapia, empleándose en muchas series como tratamiento adyuvante en estadios $\mathrm{IE}$, si bien, sí existe acuerdo en que su empleo de modo aislado no resulta eficaz.

El riesgo de recidiva es elevado, principalmente en sistema nervioso central (SNC) y teste contralateral sin olvidar otras localizaciones como la región intraocular. Uno de los predictores principales de recidiva en SNC es la combinación de niveles elevados de LDH y afectación extranodal de dos o más localizaciones. Para su prevención no existe tampoco una pauta terapéutica estan- 
darizada, aunque parece dar buenos resultados la combinación de quimioterapia intratecal y sistémica a altas dosis. Algunos autores aseguran que la combinación de tres ciclos de $\mathrm{CHOP}$, radioterapia inguinal, iliaca y de linfáticos paraaórticos así como la profilaxis de SNC con quimioterapia intratecal y radioterapia mejora la supervivencia, atribuyendo su éxito principalmente a la radioterapia regional pélvica y áreas linfáticas lumboaórticas y a la profilaxis del SNC (9). Otros abogan por la combinación de quimioterapia, profilaxis de SNC y radioterapia del teste contralateral a todos los pacientes dado el alto porcentaje de recidivas en ambas localizaciones (10). Esta falta de consenso pone de manifiesto, una vez más, la necesidad de seguir investigando en busca de pautas más efectivas y/o de nuevos tratamientos específicos.

\section{CONCLUSIÓN}

El linfoma testicular es una entidad muy poco frecuente, a pesar de ser considerado el tumor testicular más común en mayores de 60 años. En su mayoría se trata de linfomas no Hodgkin difusos de grado intermedio alto de malignidad e inmunofenotipo $B$, considerando como excepcionales los de tipo T. Su pronóstico es pobre debido a su gran tendencia a la propagación sistémica. Su tratamiento se basa en orquiectomía, quimioterapia y radioterapia, si bien no existe una pauta estandarizada del mismo.

\section{BIBLIOGRAFIAA y LECTURAS RECOMENDADAS (*lectura de interés $y^{* *}$ lectura fundamental)}

1. GREENLEE, R.; MURRAY, T.; BOLDEN, S. y cols.: "Cancer statistics 2000". CA Cancer J. Clin., 50: 7, 2000.

2. GÓMEZ GARCÍA, I.; RODRÍGUEZ PATRÓN, R.; SANZ MAYAYO, E. y cols.: "Linfoma testicular primario. Aportación de un nuevo caso y revisión de la literatura". Actas Urol. Esp., 28: 141, 2004.

3. PIEDRA LARA, J.D.; CAPITÁN MANJÓN, C.; CRUCEYRA BETRIU, G. y cols.: "Linfoma testicular primario. Presentación de un caso". Actas Urol. Esp., 26: 129, 2002.

4. PAKZAD, K.; MACLENNAN, G.T.; ELDER, J.S.: “ Follicular large cell lymphoma localized to the testis in children". J. Urol., 168: 225, 2002.

5. LEIBOVITCH, I.; GOLDWASSER, B.: "The spectrum of acquired immune deficiency syndrome-associated testicular disorders". Urology, 44: 818, 1994.

6. BRICE, P.; DE KERVILER, E.: "Lymphomas de l'appareil urogenital. Genitourinary lymphomas". Ann. Urol. (Paris), 41: 1, 2007.

7. BALLEREAU, C.; LEROY, X.; MORSCHHAUSER, F. Y cols.: "Testicular natural killer T-cell lymphoma". Int. J. Urol., 12: 223, 2005.

8. SHAHAB, N.; DOLL, D.C.: "Testicular lymphoma". Semin. Oncol., 26: 259, 1999.
9. LINASSIER, C.; DESABLENS, B.; LEFRANCQ, T. y cols.: "Stage I-IIE primary non-Hodgkin's lymphoma of the testis: Results of a prospective trial by the GOELAMS Study Group". Clin. Lymphoma, 3: 167, 2002.

10. KONDO, T.; WADA, H.; YATA, K.: "Seven patients with stage I and II primary testicular lymphoma". Rinsho Ketrueki, 43: 473, 2002.

Casos Clínicos

Arch. Esp. Urol., 61, 4 (53 1-533), 2008

\section{METÁSTASIS TESTICULAR METACRÓNICA SECUNDARIA A ADENOCARCINOMA RENAL DE CÉLULAS CLARAS}

\author{
Roberto Llarena Ibarguren, Jorge García-Olaverri \\ Rodríguez, lgor Azurmendi Arin, Ivan Olano Grasa y \\ Carlos Pertusa Peña.
}

Servicio de Urología. Hospital de Cruces. Baracaldo. Vizcaya. España.

Resumen.- OBJETIVO: Presentar un caso de metástasis inusual en testículo de origen renal.

MÉTODO: Paciente de 57 años afecto y en tratamiento por un cáncer renal derecho diseminado que durante su evolución con terapia médica aparecieron metástasis óseas y un nódulo doloroso en polo superior testicular derecho, que obligó a cirugía, con resultado de metástasis de adenocarcinoma de células claras.

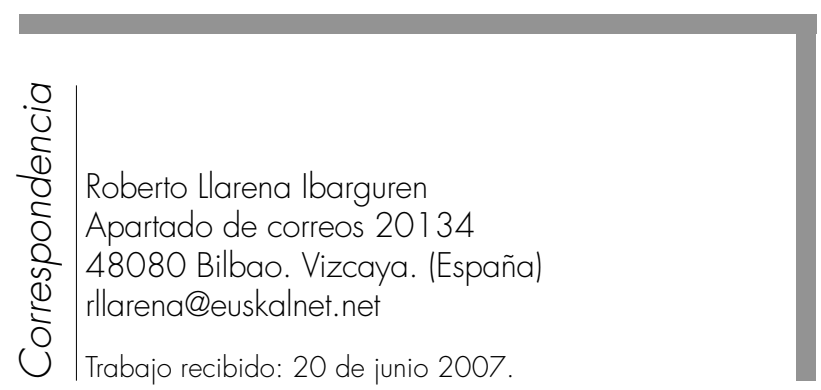

